\title{
Author Correction: Magnetic skyrmion bundles and their current-driven dynamics
}

Jin Tang (D), Yaodong Wu, Weiwei Wang (D), Lingyao Kong, Boyao Lv, Wensen Wei, Jiadong Zang, Mingliang Tian and Haifeng Du (1)

Correction to: Nature Nanotechnology https://doi.org/10.1038/s41565-021-00954-9, published online 2 August 2021

In the version of this Article initially published, the Acknowledgements text was missing this last sentence: "J.Z. was supported by U.S. Department of Energy, Office of Science, Basic Energy Sciences (grant no. DE-SC0020221)."

The original Article has been corrected in the online and in print versions of the paper.

Published online: 3 September 2021

https://doi.org/10.1038/s41565-021-00985-2

(C) The Author(s), under exclusive licence to Springer Nature Limited 2021 